\title{
Generative Adversarial Networks for Pre-training of Medical Image Segmentation Networks
}

\section{CURRENT STATUS: POSTED}

Research Square

\section{Kun Chen}

Fudan University School of Basic Medical Sciences

kchen17@fudan.edu.cnCorresponding Author

ORCiD: https://orcid.org/0000-0001-9172-4562

Manning Wang

Fudan University School of Basic Medical Sciences

Zhijian Song

Fudan University School of Basic Medical Sciences

\section{DOI:}

10.21203/rs.2.22269/v1

\section{SUBJECT AREAS}

Biomedical Engineering

\section{KEYWORDS}

Generative Adversarial Networks, Segmentation networks, Medical image 29 segmentation, Preliminary training 
Abstract

Background: Deep neural networks have been widely used in medical image segmentation and have achieved state-of-the-art performance in many tasks. However, different from the segmentation of natural images or video frames, the manual segmentation of anatomical structures in medical images needs high expertise so the scale of labeled training data is very small, which is a major obstacle for the improvement of deep neural networks performance in medical image segmentation.

Methods: In this paper, we proposed a new end-to-end generation-segmentation framework by integrating Generative Adversarial Networks (GAN) and a segmentation network and train them simultaneously. The novelty is that during the training of the GAN, the intermediate synthetic images generated by the generator of the GAN are used to pre-train the segmentation network. As the advances of the training of the GAN, the synthetic images evolve gradually from being very coarse to containing more realistic textures, and these images help train the segmentation network gradually. After the training of GAN, the segmentation network is then fine-tuned by training with the real labeled images.

Results: We evaluated the proposed framework on four different datasets, including 2D cardiac dataset and lung dataset, 3D prostate dataset and liver dataset. Compared with original U-net and CE-Net, our framework can achieve better segmentation performance. Our framework also can get better segmentation results than U-net on small datasets. In addition, our framework is more effective than the usual data augmentation methods.

Conclusions: The proposed framework can be used as a pre-train method of segmentation network, which helps to get a better segmentation result. Our method can solve the shortcomings of current data augmentation methods to some extent.

Full Text

Due to technical limitations, full-text HTML conversion of this manuscript could not be completed. However, the manuscript can be downloaded and accessed as a PDF.

Figures 

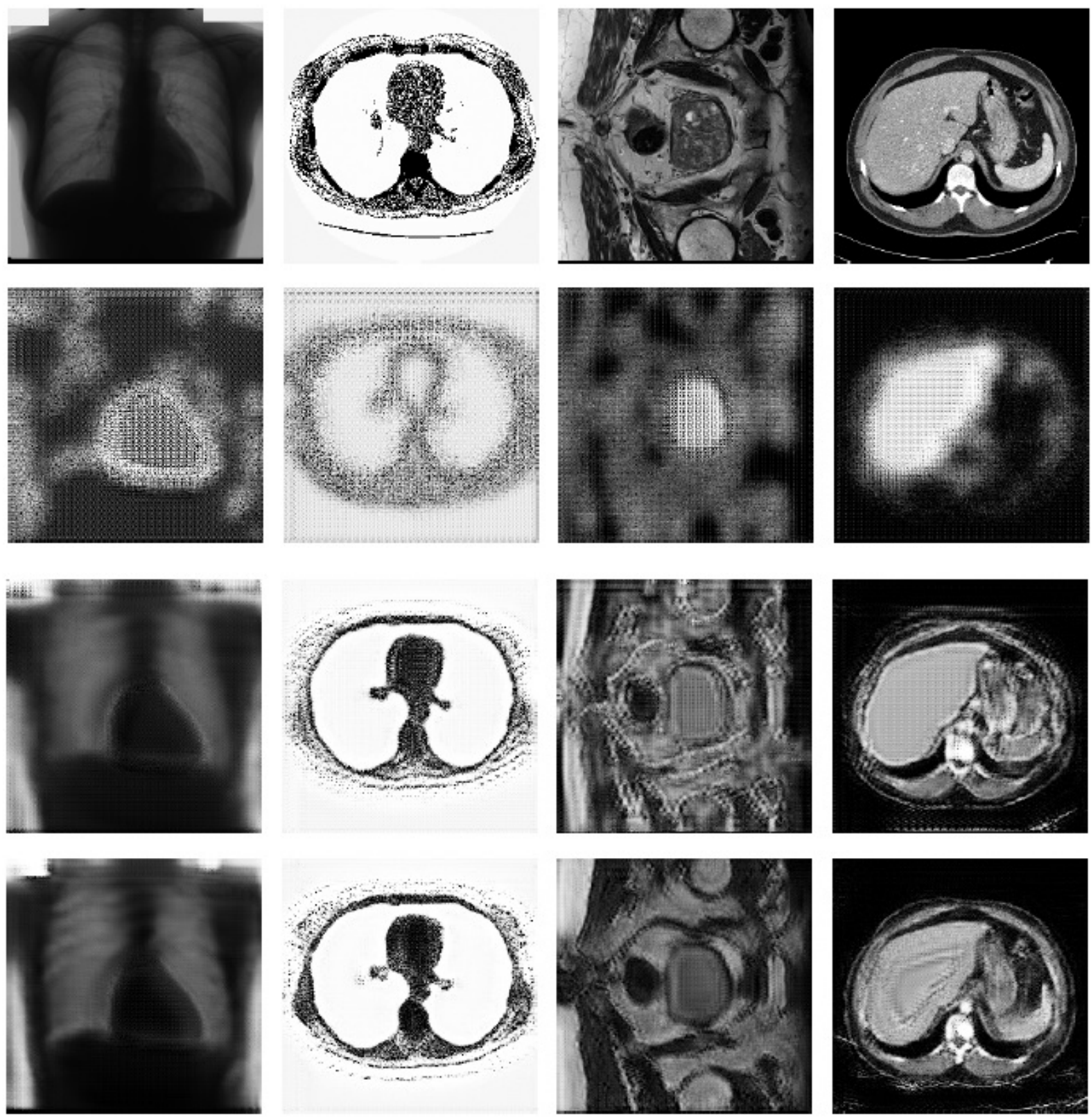

Cardiac

LUNA

Prostate

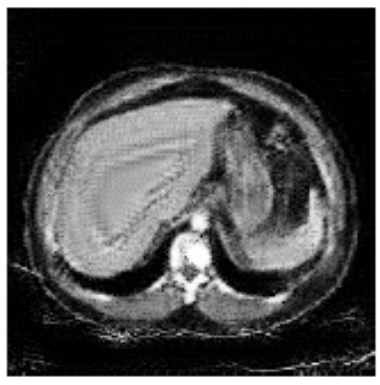

Liver

Figure 1

Generated images by the generator of GAN in different iterations for different datasets. The first row represents the original images, and the 2 nd, 3rd and fourth rows represent the generated images after 1, 50, and 100 epochs, respectively. 

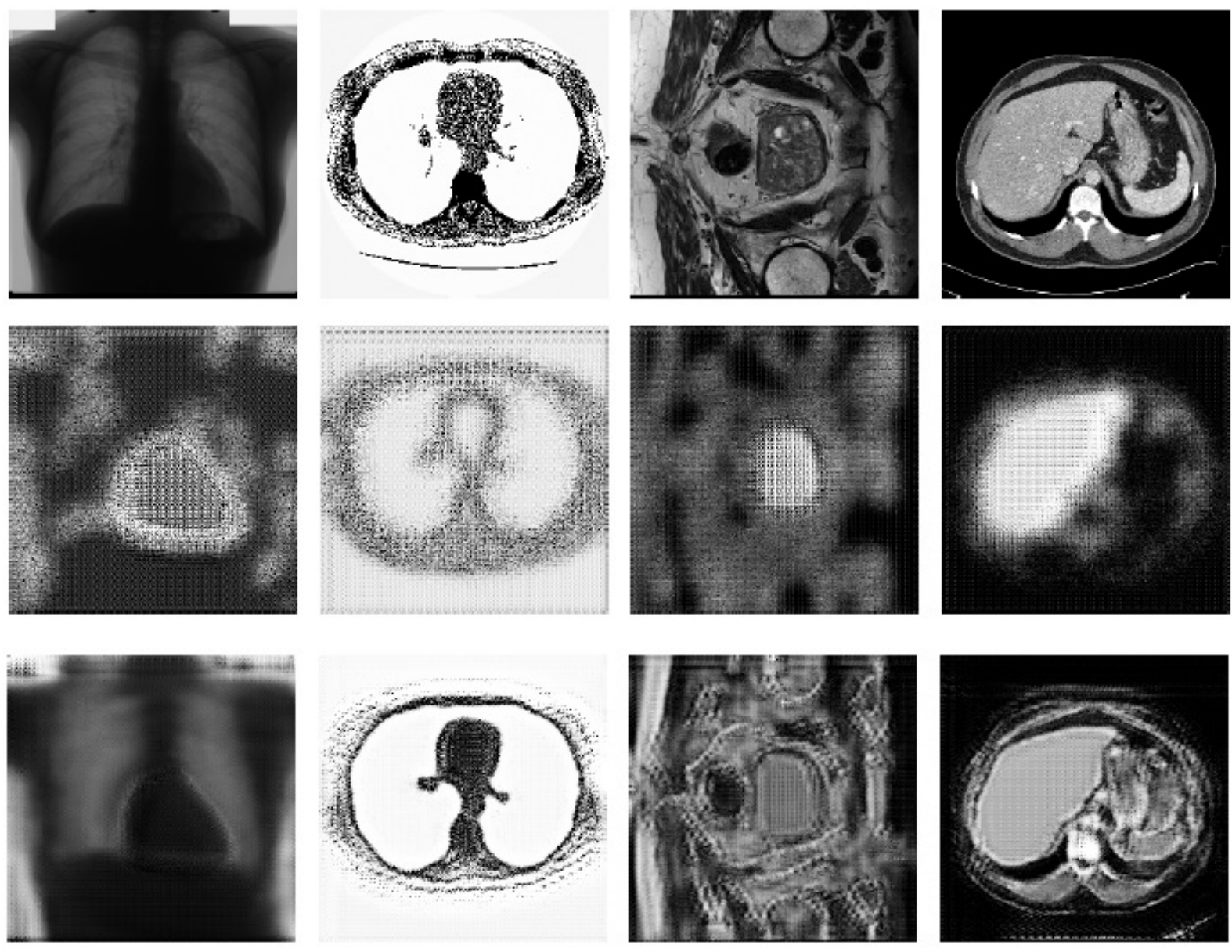

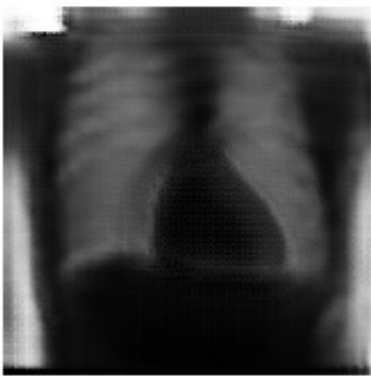

Cardiac

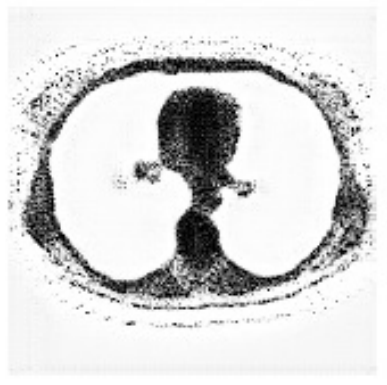

LUNA

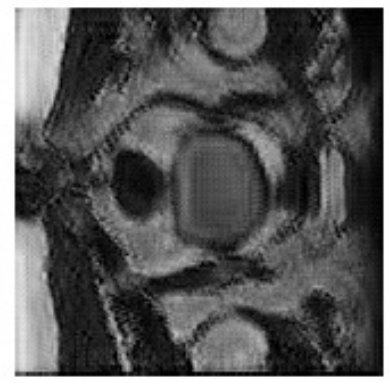

Prostate

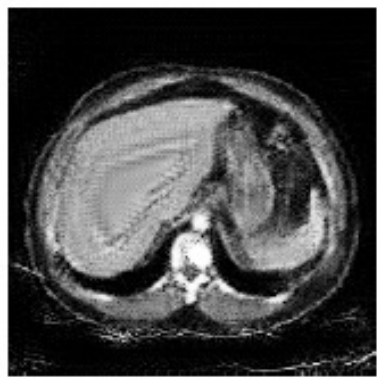

Liver

Figure 1

Generated images by the generator of GAN in different iterations for different datasets. The first row represents the original images, and the 2 nd, 3rd and fourth rows represent the generated images after 1,50 , and 100 epochs, respectively. 


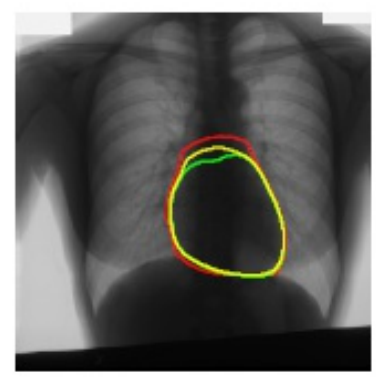

(a)

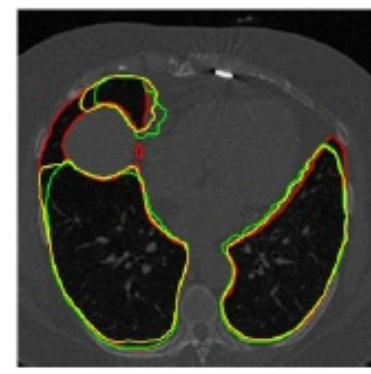

(b)

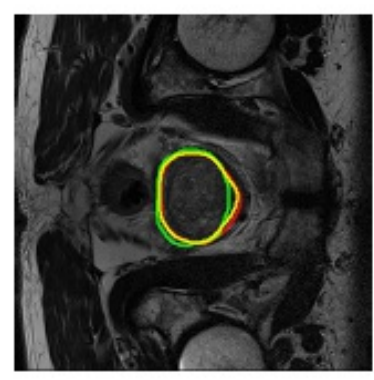

(c)

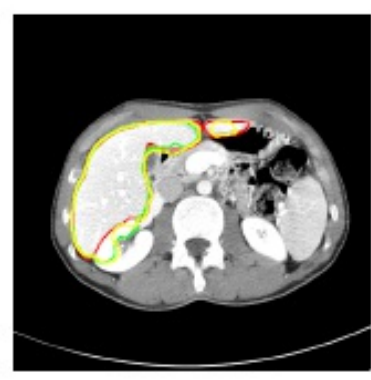

(d)

Figure 2

Segmentation results in different datasets. (a) cardiac segmentation results, (b) LUNA segmentation results, (c) prostate segmentation results, (d) liver segmentation results, where red is the gold standard, green is the U-net segmentation results, and yellow is our segmentation results.

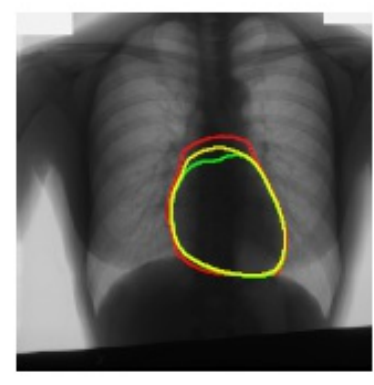

(a)

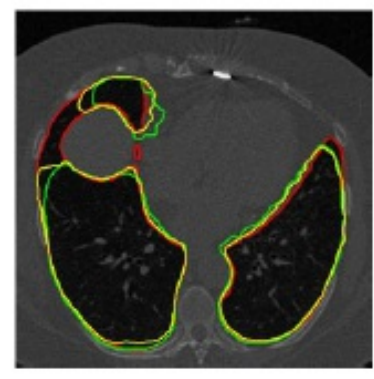

(b)

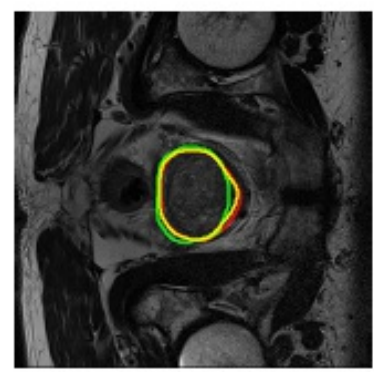

(c)

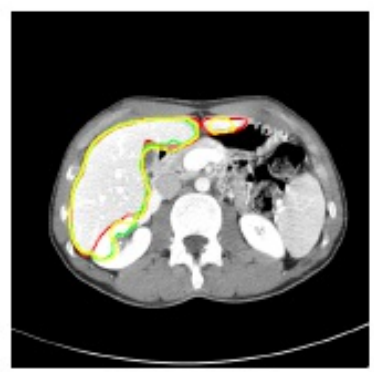

(d)

Figure 2

Segmentation results in different datasets. (a) cardiac segmentation results, (b) LUNA segmentation results, (c) prostate segmentation results, (d) liver segmentation results, where red is the gold standard, green is the U-net segmentation results, and yellow is our segmentation results. 


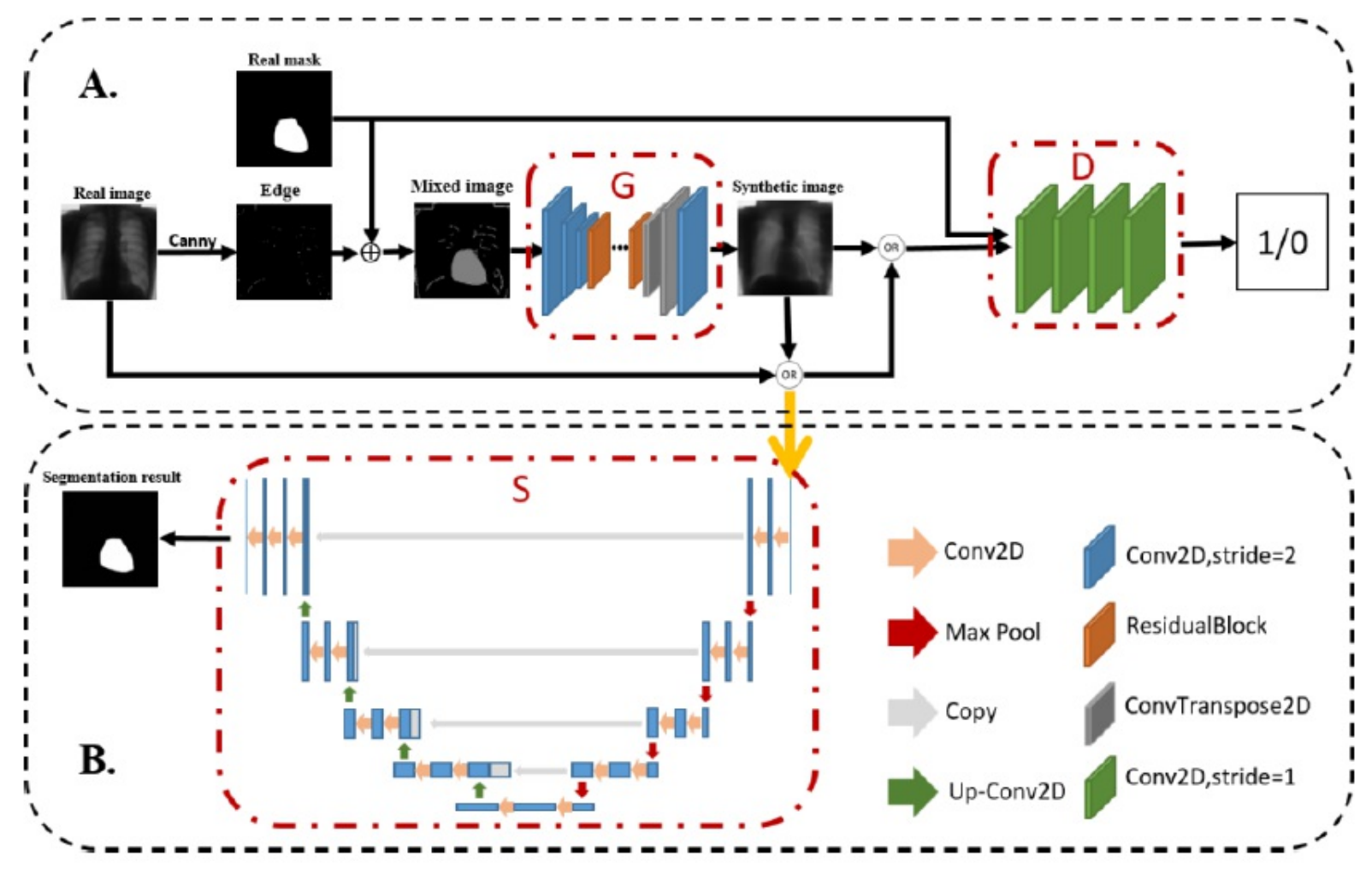

Figure 3

The structure of our proposed framework. (A)The data synthesis part. G represents the Generator of GAN, D represents the Discriminator of GAN. (B)The segmentation part. S represents the segmentation network, such as U-net. 


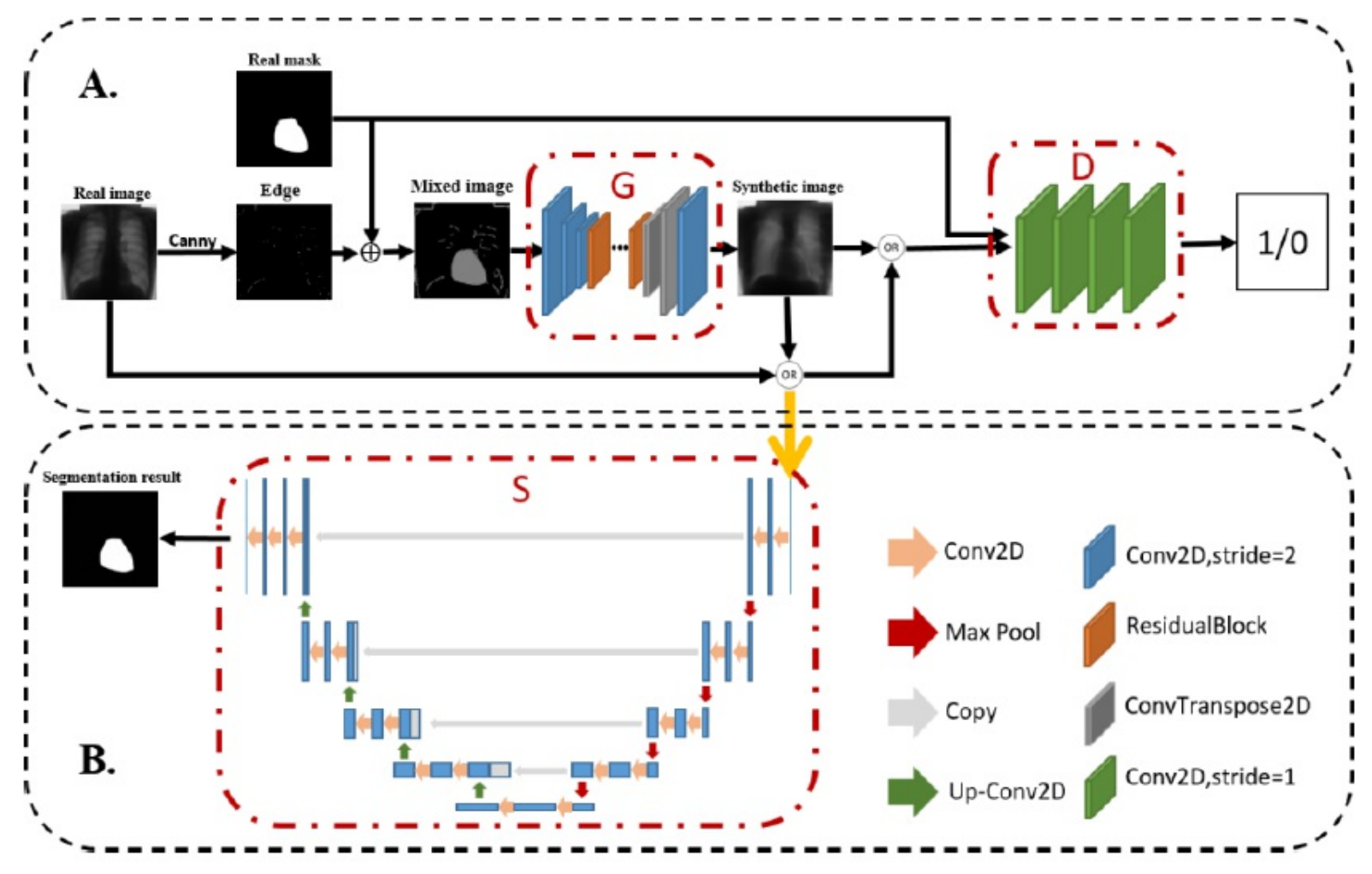

Figure 3

The structure of our proposed framework. (A)The data synthesis part. G represents the Generator of GAN, D represents the Discriminator of GAN. (B)The segmentation part. S represents the segmentation network, such as U-net. 